Acta Crystallographica Section E

Structure Reports

Online

ISSN 1600-5368

\section{\{6,6'-Dimethoxy-2,2'-[4,5-dimethyl-o- phenylenebis(nitrilomethylidyne)]- diphenolato\}nickel(II)}

\section{Hadi Kargar, ${ }^{a}$ Reza Kia, ${ }^{b, c} \neq$ Muhammad Nawaz Tahir ${ }^{d} *$ and Atefeh Sahraei ${ }^{\mathrm{a}}$}

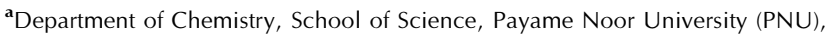
Ardakan, Yazd, Iran, ${ }^{\mathbf{b}}$ Department of Chemistry, Science and Research Branch, Islamic Azad University, Tehran, Iran, ' X-ray Crystallography Lab., Plasma Physics Research Center, Science and Research Branch, Islamic Azad University, Tehran, Iran, and ${ }^{\mathbf{d}}$ Department of Physics, University of Sargodha, Punjab, Pakistan

Correspondence e-mail: dmntahir_uos@yahoo.com

Received 4 September 2010; accepted 7 September 2010

Key indicators: single-crystal X-ray study; $T=296 \mathrm{~K}$; mean $\sigma(\mathrm{C}-\mathrm{C})=0.005 \AA$; $R$ factor $=0.047 ; w R$ factor $=0.124 ;$ data-to-parameter ratio $=18.1$.

In the title Schiff base complex, $\left[\mathrm{Ni}\left(\mathrm{C}_{24} \mathrm{H}_{22} \mathrm{~N}_{2} \mathrm{O}_{4}\right)\right]$, the $\mathrm{Ni}^{\mathrm{II}}$ atom has a slightly distorted square-planar coordination environment. The dihedral angles between the central benzene ring and the two outer rings are 7.62 (16) and $9.78(17)^{\circ}$. The crystal structure is stabilized by intermolecular $\mathrm{C}-\mathrm{H} \cdots \mathrm{O}$ hydrogen bonds and $\pi-\pi$ interactions with a centroid-centroid distance of 3.8218 (19) $\AA$.

\section{Related literature}

For background to Schiff base-metal complexes, see: Granovski et al. (1993); Blower et al. (1998); Elmali et al. (2000). For standard values of bond lengths, see: Allen et al. (1987).

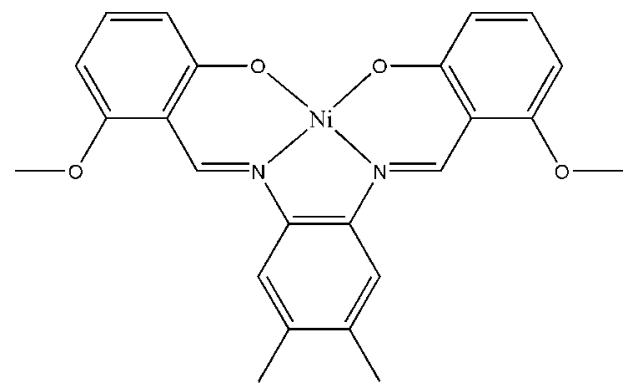

\section{Experimental}

Crystal data

$\left[\mathrm{Ni}\left(\mathrm{C}_{24} \mathrm{H}_{22} \mathrm{~N}_{2} \mathrm{O}_{4}\right)\right]$

$M_{r}=461.15$

Monoclinic, $P 2_{1} / n$

$a=12.8057$ (6) $\AA$

$b=12.6514(5) \AA$

$c=13.0263(6) \AA$

$\beta=101.730(2)^{\circ}$

$V=2066.32(16) \AA^{3}$

$Z=4$

Mo $K \alpha$ radiation

$\mu=0.97 \mathrm{~mm}^{-1}$

$T=296 \mathrm{~K}$

$0.24 \times 0.14 \times 0.08 \mathrm{~mm}$

\section{Data collection}

Bruker SMART APEXII CCD area-detector diffractometer

Absorption correction: multi-scan (SADABS; Bruker, 2005)

$T_{\text {min }}=0.800, T_{\max }=0.926$

Refinement

$R\left[F^{2}>2 \sigma\left(F^{2}\right)\right]=0.047$

$w R\left(F^{2}\right)=0.124$

$S=1.03$

5146 reflections

36150 measured reflections 5146 independent reflections 3179 reflections with $I>2 \sigma(I)$ $R_{\text {int }}=0.068$

Table 1

Hydrogen-bond geometry $\left(\AA{ }^{\circ}\right)$.

\begin{tabular}{lllll}
\hline$D-\mathrm{H} \cdots A$ & $D-\mathrm{H}$ & $\mathrm{H} \cdots A$ & $D \cdots A$ & $D-\mathrm{H} \cdots A$ \\
\hline $\mathrm{C} 7-\mathrm{H} 7 C \cdots \mathrm{O}^{\mathrm{i}}$ & 0.96 & 2.51 & $3.424(5)$ & 158 \\
$\mathrm{C} 21-\mathrm{H} 21 \cdots \mathrm{O} 2^{\mathrm{ii}}$ & 0.93 & 2.52 & $3.340(4)$ & 147 \\
\hline
\end{tabular}

Symmetry codes: (i) $-x+\frac{1}{2}, y+\frac{1}{2},-z+\frac{1}{2}$; (ii) $x-\frac{1}{2},-y+\frac{3}{2}, z+\frac{1}{2}$.

Data collection: APEX2 (Bruker, 2005); cell refinement: SAINT (Bruker, 2005); data reduction: $S A I N T$; $\operatorname{program}(\mathrm{s})$ used to solve structure: SHELXS97 (Sheldrick, 2008); program(s) used to refine structure: SHELXL97 (Sheldrick, 2008); molecular graphics: SHELXTL (Sheldrick, 2008); software used to prepare material for publication: SHELXTL and PLATON (Spek, 2009).

HK and AS thank PNU for financial support. RK thanks the Islamic Azad University. MNT thanks the University of Sargodha, Pakistan, for the research facilities.

Supplementary data and figures for this paper are available from the IUCr electronic archives (Reference: SU2210).

\section{References}

Allen, F. H., Kennard, O., Watson, D. G., Brammer, L., Orpen, A. G. \& Taylor, R. (1987). J. Chem. Soc. Perkin Trans. 2, pp. S1-19.

Blower, P. J. (1998). Transition Met. Chem. 23, 109-112.

Bruker (2005). APEX2, SAINT and SADABS. Bruker AXS Inc., Madison, Wisconsin, USA.

Elmali, A., Elerman, Y. \& Svoboda, I. (2000). Acta Cryst. C56, 423-424.

Granovski, A. D., Nivorozhkin, A. L. \& Minkin, V. I. (1993). Coord. Chem. Rev. 126, 1-69.

Sheldrick, G. M. (2008). Acta Cryst. A64, 112-122.

Spek, A. L. (2009). Acta Cryst. D65, 148-155. 


\section{supporting information}

Acta Cryst. (2010). E66, m1246 [doi:10.1107/S1600536810035890]

\section{\{6,6'-Dimethoxy-2,2'-[4,5-dimethyl-o-phenylenebis(nitrilomethyl- idyne)]diphenolato\}nickel(II)}

\section{Hadi Kargar, Reza Kia, Muhammad Nawaz Tahir and Atefeh Sahraei}

\section{S1. Comment}

Schiff base complexes are one of the most important stereochemical models in transition metal coordination chemistry, with ease of preparation and structural variations (Granovski et al., 1993). Metal derivatives of the Schiff bases have been studied extensively, and nickel(II) and copper(II) complexes play a major role in both synthetic and structurel research (Elmali et al., 2000; Blower et al., 1998).

The molecular structure of the title molecule is illustrated in Fig. 1. The bond lengths (Allen et al., 1987) and angles are within normal ranges. The geometry around the $\mathrm{Ni}^{\mathrm{II}}$ atom is square-planar being coordinated by the $\mathrm{N}_{2} \mathrm{O}_{2}$ donor atoms of the tetradenate Schiff base ligand. The dihedral angle between the mean planes of the central aromatic ring (C9-C14) with the two outer rings (C1-C6 and C18-C23) are $7.62(16)$ and $9.78(17)^{\circ}$, respectively.

The crystal structure is stabilized by intermolecular $\mathrm{C}-\mathrm{H} \cdots \mathrm{O}$ hydrogen bonds (Table 1 ) and $\pi-\pi$ interactions

$\left[\mathrm{Cg} 1 \cdots \mathrm{Cg} 2^{\mathrm{i}}=3.8218(19) \AA ; \mathrm{Cg} 1\right.$ and $\mathrm{Cg} 2$ are the centroids of the $\mathrm{C} 1-\mathrm{C} 6$ and $\mathrm{C} 9-\mathrm{C} 14$ rings, respectively; symmetry code (i) $-\mathrm{x}, 2-\mathrm{y},-\mathrm{z}]$.

\section{S2. Experimental}

The title compound was synthesized by adding bis(6-methoxysalicylidene)-4,5-dimethyl phenylenediamine ( $2 \mathrm{mmol})$ to a solution of $\mathrm{NiCl}_{2} \cdot 6 \mathrm{H}_{2} \mathrm{O}(2 \mathrm{mmol})$ in ethanol $(30 \mathrm{ml})$. The mixture was refluxed with stirring for $30 \mathrm{~min}$. The resultant red solution was filtered. Dark-red plate-like single crystals of the title compound, suitable for $X$-ray structure analysis, were obtained by slow evaporation at RT of a solution in ethanol over a period of several days.

\section{S3. Refinement}

All the $\mathrm{H}$-atoms were positioned geometrically and included in a riding model approximation: $\mathrm{C}-\mathrm{H}=0.93$ and $0.96 \AA$ for $\mathrm{CH}$ and $\mathrm{CH}_{3} \mathrm{H}$-atoms, respectively, with $\mathrm{U}_{\text {iso }}(\mathrm{H})=\mathrm{k} \times \mathrm{U}_{\mathrm{eq}}(\mathrm{C})$, where $\mathrm{k}=1.5$ for methyl $\mathrm{H}$-atoms and $\mathrm{k}=1.2$ for all other H-atoms. 


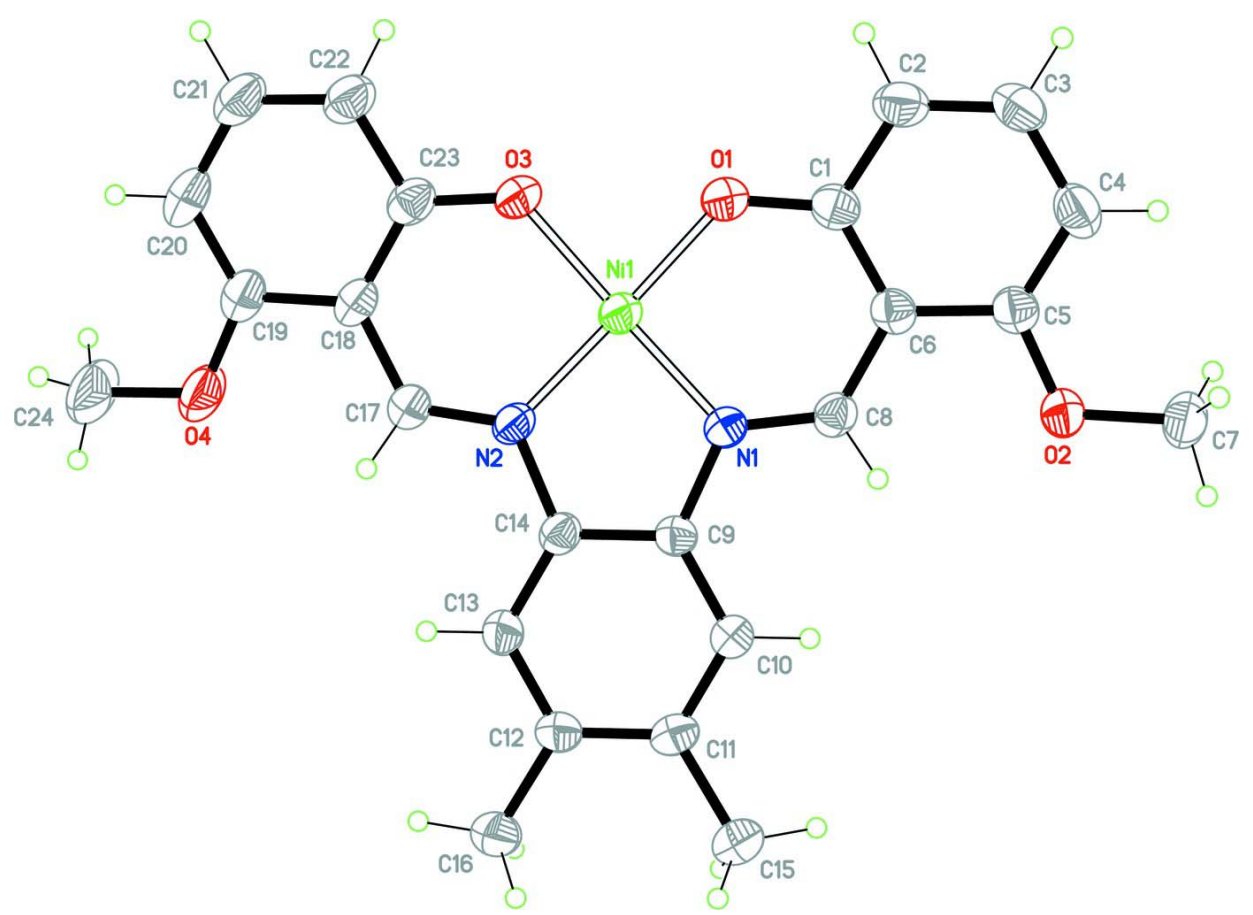

\section{Figure 1}

The molecular structure of the title molecule, showing $40 \%$ probability displacement ellipsoids and the atom numbering scheme. 


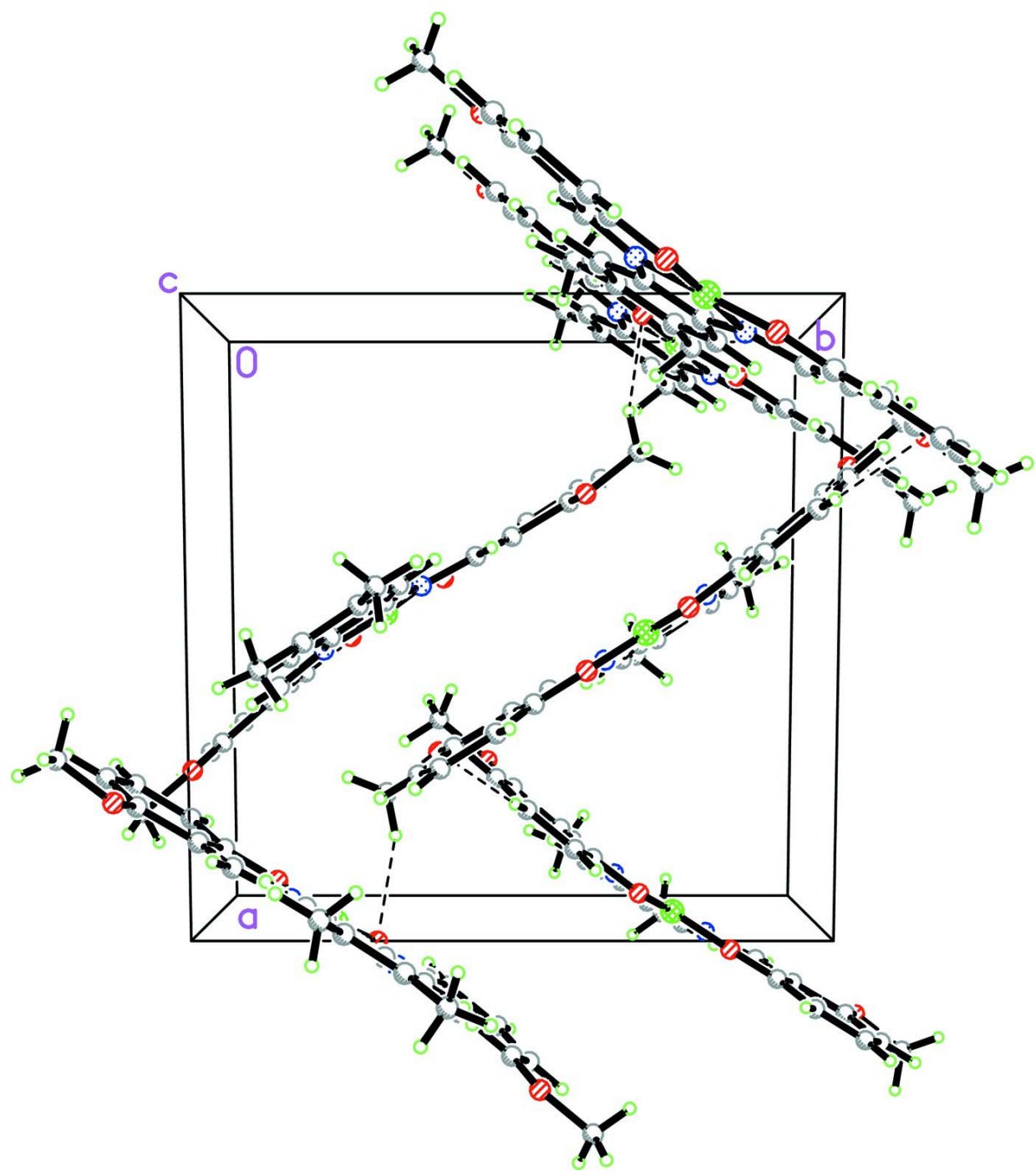

Figure 2

The crystal packing diagram of the title compound viewed down the $c$-axis showing intermolecular interactions as dashed lines.

\section{\{6,6'-Dimethoxy-2,2'-[4,5-dimethyl-o- phenylenebis(nitrilomethylidyne)]diphenolato\}nickel(II)}

\section{Crystal data}

$\left[\mathrm{Ni}\left(\mathrm{C}_{24} \mathrm{H}_{22} \mathrm{~N}_{2} \mathrm{O}_{4}\right)\right]$

$M_{r}=461.15$

Monoclinic, $P 2_{1} / n$

Hall symbol: -P 2 yn

$a=12.8057$ (6) $\AA$

$b=12.6514(5) \AA$

$c=13.0263(6) \AA$

$\beta=101.730(2)^{\circ}$

$V=2066.32(16) \AA^{3}$

$Z=4$
$F(000)=960$

$D_{\mathrm{x}}=1.482 \mathrm{Mg} \mathrm{m}^{-3}$

Mo $K \alpha$ radiation, $\lambda=0.71073 \AA$

Cell parameters from 2525 reflections

$\theta=2.5-29.5^{\circ}$

$\mu=0.97 \mathrm{~mm}^{-1}$

$T=296 \mathrm{~K}$

Plate, red

$0.24 \times 0.14 \times 0.08 \mathrm{~mm}$ 


\section{Data collection}

Bruker SMART APEXII CCD area-detector diffractometer

Radiation source: fine-focus sealed tube Graphite monochromator

$\varphi$ and $\omega$ scans

Absorption correction: multi-scan

(SADABS; Bruker, 2005)

$T_{\min }=0.800, T_{\max }=0.926$

\section{Refinement}

Refinement on $F^{2}$

Least-squares matrix: full

$R\left[F^{2}>2 \sigma\left(F^{2}\right)\right]=0.047$

$w R\left(F^{2}\right)=0.124$

$S=1.03$

5146 reflections

284 parameters

0 restraints

Primary atom site location: structure-invariant direct methods
36150 measured reflections

5146 independent reflections

3179 reflections with $I>2 \sigma(I)$

$R_{\text {int }}=0.068$

$\theta_{\max }=28.4^{\circ}, \theta_{\min }=2.0^{\circ}$

$h=-17 \rightarrow 17$

$k=-16 \rightarrow 16$

$l=-17 \rightarrow 17$

Secondary atom site location: difference Fourier map

Hydrogen site location: inferred from

neighbouring sites

$\mathrm{H}$-atom parameters constrained

$w=1 /\left[\sigma^{2}\left(F_{\mathrm{o}}^{2}\right)+(0.0484 P)^{2}+1.1318 P\right]$

where $P=\left(F_{\mathrm{o}}{ }^{2}+2 F_{\mathrm{c}}{ }^{2}\right) / 3$

$(\Delta / \sigma)_{\max }<0.001$

$\Delta \rho_{\max }=0.48 \mathrm{e} \AA^{-3}$

$\Delta \rho_{\min }=-0.34$ e $\AA^{-3}$

Special details

Geometry. All esds (except the esd in the dihedral angle between two 1.s. planes) are estimated using the full covariance matrix. The cell esds are taken into account individually in the estimation of esds in distances, angles and torsion angles; correlations between esds in cell parameters are only used when they are defined by crystal symmetry. An approximate (isotropic) treatment of cell esds is used for estimating esds involving l.s. planes.

Refinement. Refinement of $\mathrm{F}^{2}$ against ALL reflections. The weighted R-factor $\mathrm{wR}$ and goodness of fit $\mathrm{S}$ are based on $\mathrm{F}^{2}$, conventional R-factors $R$ are based on $F$, with $F$ set to zero for negative $F^{2}$. The threshold expression of $F^{2}>2 \operatorname{sigma}\left(F^{2}\right)$ is used only for calculating R-factors(gt) etc. and is not relevant to the choice of reflections for refinement. R-factors based on $\mathrm{F}^{2}$ are statistically about twice as large as those based on F, and R- factors based on ALL data will be even larger.

Fractional atomic coordinates and isotropic or equivalent isotropic displacement parameters $\left(\AA^{2}\right)$

\begin{tabular}{lllll}
\hline & $x$ & $y$ & $z$ & $U_{\text {iso }} * / U_{\text {eq }}$ \\
\hline Ni1 & $0.01614(3)$ & $0.78355(3)$ & $0.17262(3)$ & $0.03619(14)$ \\
O1 & $0.07749(19)$ & $0.88142(16)$ & $0.27017(17)$ & $0.0481(6)$ \\
O2 & $0.21622(19)$ & $1.12212(16)$ & $0.05147(18)$ & $0.0483(6)$ \\
O3 & $-0.02481(18)$ & $0.72048(16)$ & $0.28477(17)$ & $0.0453(5)$ \\
O4 & $-0.2538(2)$ & $0.4558(2)$ & $0.1089(2)$ & $0.0768(9)$ \\
N1 & $0.06210(19)$ & $0.84778(18)$ & $0.06261(19)$ & $0.0346(6)$ \\
N2 & $-0.0465(2)$ & $0.68259(19)$ & $0.07723(19)$ & $0.0364(6)$ \\
C1 & $0.1314(3)$ & $0.9655(2)$ & $0.2565(3)$ & $0.0410(7)$ \\
C2 & $0.1766(3)$ & $1.0258(3)$ & $0.3455(3)$ & $0.0506(9)$ \\
H2 & 0.1675 & 1.0047 & 0.4115 & $0.061^{*}$ \\
C3 & $0.2336(3)$ & $1.1150(3)$ & $0.3353(3)$ & $0.0528(9)$ \\
H3 & 0.2638 & 1.1528 & 0.3953 & $0.063^{*}$ \\
C4 & $0.2480(3)$ & $1.1515(3)$ & $0.2381(3)$ & $0.0506(9)$ \\
H4 & 0.2863 & 1.2131 & 0.2332 & $0.061^{*}$ \\
C5 & $0.2054(3)$ & $1.0954(2)$ & $0.1504(3)$ & $0.0398(7)$ \\
C6 & $0.1472(2)$ & $0.9993(2)$ & $0.1570(2)$ & $0.0365(7)$
\end{tabular}




$\begin{array}{lllll}\text { C7 } & 0.2852(3) & 1.2086(3) & 0.0418(3) & 0.0536(9) \\ \text { H7A } & 0.2584 & 1.2717 & 0.0683 & 0.080^{*} \\ \text { H7B } & 0.2880 & 1.2184 & -0.0307 & 0.080^{*} \\ \text { H7C } & 0.3555 & 1.1939 & 0.0813 & 0.080^{*} \\ \text { C8 } & 0.1120(2) & 0.9388(2) & 0.0659(2) & 0.0367(7) \\ \text { H8 } & 0.1252 & 0.9652 & 0.0031 & 0.044^{*} \\ \text { C9 } & 0.0357(2) & 0.7889(2) & -0.0328(2) & 0.0349(7) \\ \text { C10 } & 0.0623(3) & 0.8146(2) & -0.1274(2) & 0.0398(7) \\ \text { H10 } & 0.1035 & 0.8743 & -0.1319 & 0.048^{*} \\ \text { C11 } & 0.0285(3) & 0.7528(3) & -0.2154(3) & 0.0417(7) \\ \text { C12 } & -0.0325(3) & 0.6620(2) & -0.2082(2) & 0.0407(7) \\ \text { C13 } & -0.0573(3) & 0.6355(2) & -0.1132(3) & 0.0421(8) \\ \text { H13 } & -0.0966 & 0.5746 & -0.1080 & 0.050^{*} \\ \text { C14 } & -0.0241(2) & 0.6985(2) & -0.0250(2) & 0.0347(7) \\ \text { C15 } & 0.0579(3) & 0.7840(3) & -0.3177(3) & 0.0583(10) \\ \text { H15A } & 0.0972 & 0.8491 & -0.3086 & 0.087^{*} \\ \text { H15B } & -0.0058 & 0.7932 & -0.3703 & 0.087^{*} \\ \text { H15C } & 0.1010 & 0.7295 & -0.3394 & 0.087^{*} \\ \text { C16 } & -0.0759(3) & 0.5964(3) & -0.3041(3) & 0.0534(9) \\ \text { H16A } & -0.1054 & 0.5320 & -0.2833 & 0.080^{*} \\ \text { H16B } & -0.0194 & 0.5800 & -0.3399 & 0.080^{*} \\ \text { H16C } & -0.1306 & 0.6353 & -0.3501 & 0.080^{*} \\ \text { C17 } & -0.1087(3) & 0.6065(2) & 0.0954(2) & 0.0405(7) \\ \text { H17 } & -0.1382 & 0.5632 & 0.0393 & 0.049^{*} \\ \text { C18 } & -0.1353(3) & 0.5845(2) & 0.1932(3) & 0.0406(7) \\ \text { C19 } & -0.2085(3) & 0.5008(3) & 0.2020(3) & 0.0520(9) \\ \text { C20 } & -0.2290(3) & 0.4723(3) & 0.2968(3) & 0.0583(10) \\ \text { H20 } & -0.2767 & 0.4180 & 0.3016 & 0.070^{*} \\ \text { C21 } & -0.1776(3) & 0.5255(3) & 0.3863(3) & 0.0554(10) \\ \text { H21 } & -0.1906 & 0.5049 & 0.4511 & 0.067^{*} \\ \text { C22 } & -0.1088(3) & 0.6069(3) & 0.3820(3) & 0.0514(9) \\ \text { H22 } & -0.0758 & 0.6408 & 0.4435 & 0.062^{*} \\ \text { C23 } & -0.0873(3) & 0.6399(2) & 0.2851(3) & 0.0420(8) \\ \text { C24 } & -0.3272(5) & 0.3705(4) & 0.1122(4) & 0.107(2) \\ \text { H24A } & -0.2894 & 0.3109 & 0.1473 & 0.161^{*} \\ \text { H24B } & -0.3606 & 0.3507 & 0.0420 & 0.161^{*} \\ \text { H24C } & -0.3808 & 0.3928 & 0.1495 & 0.161^{*} \\ & & & & \end{array}$

Atomic displacement parameters $\left(\AA^{2}\right)$

\begin{tabular}{lllllll}
\hline & $U^{11}$ & $U^{22}$ & $U^{33}$ & $U^{12}$ & $U^{13}$ & $U^{23}$ \\
\hline Ni1 & $0.0430(3)$ & $0.0344(2)$ & $0.0338(2)$ & $-0.00055(18)$ & $0.01401(17)$ & $-0.00004(17)$ \\
O1 & $0.0676(16)$ & $0.0428(12)$ & $0.0370(13)$ & $-0.0083(11)$ & $0.0177(12)$ & $-0.0029(10)$ \\
O2 & $0.0570(15)$ & $0.0408(12)$ & $0.0511(15)$ & $-0.0117(11)$ & $0.0203(12)$ & $-0.0070(10)$ \\
O3 & $0.0599(14)$ & $0.0443(12)$ & $0.0366(12)$ & $-0.0058(11)$ & $0.0210(11)$ & $0.0005(10)$ \\
O4 & $0.096(2)$ & $0.0802(18)$ & $0.0535(17)$ & $-0.0504(17)$ & $0.0144(16)$ & $0.0106(15)$ \\
N1 & $0.0350(14)$ & $0.0370(13)$ & $0.0337(14)$ & $-0.0019(10)$ & $0.0117(11)$ & $-0.0013(11)$ \\
N2 & $0.0425(15)$ & $0.0360(12)$ & $0.0342(14)$ & $-0.0006(11)$ & $0.0158(12)$ & $0.0034(11)$
\end{tabular}




$\begin{array}{lllllll}\text { C1 } & 0.0447(19) & 0.0377(16) & 0.0426(19) & 0.0038(14) & 0.0134(15) & -0.0060(14) \\ \text { C2 } & 0.066(2) & 0.0485(19) & 0.038(2) & 0.0014(17) & 0.0135(18) & -0.0058(15) \\ \text { C3 } & 0.058(2) & 0.053(2) & 0.045(2) & -0.0070(17) & 0.0061(18) & -0.0139(17) \\ \text { C4 } & 0.052(2) & 0.0418(17) & 0.058(2) & -0.0081(15) & 0.0113(18) & -0.0122(16) \\ \text { C5 } & 0.0378(18) & 0.0377(16) & 0.046(2) & 0.0018(13) & 0.0135(15) & -0.0065(14) \\ \text { C6 } & 0.0364(17) & 0.0322(14) & 0.0420(19) & 0.0041(12) & 0.0108(14) & -0.0040(13) \\ \text { C7 } & 0.055(2) & 0.0467(18) & 0.064(2) & -0.0115(17) & 0.0230(19) & -0.0006(17) \\ \text { C8 } & 0.0363(17) & 0.0362(15) & 0.0401(18) & 0.0016(13) & 0.0140(14) & 0.0005(13) \\ \text { C9 } & 0.0370(17) & 0.0349(14) & 0.0345(16) & -0.0018(13) & 0.0114(13) & -0.0044(13) \\ \text { C10 } & 0.0441(19) & 0.0398(16) & 0.0375(18) & -0.0058(14) & 0.0129(15) & -0.0011(13) \\ \text { C11 } & 0.0430(19) & 0.0481(17) & 0.0375(18) & 0.0019(14) & 0.0160(15) & -0.0002(14) \\ \text { C12 } & 0.0450(19) & 0.0423(17) & 0.0376(18) & -0.0009(14) & 0.0148(15) & -0.0058(14) \\ \text { C13 } & 0.045(2) & 0.0382(16) & 0.047(2) & -0.0091(14) & 0.0181(16) & -0.0056(14) \\ \text { C14 } & 0.0340(17) & 0.0364(15) & 0.0358(17) & -0.0010(12) & 0.0117(13) & 0.0018(12) \\ \text { C15 } & 0.070(2) & 0.065(2) & 0.045(2) & -0.015(2) & 0.0241(19) & -0.0027(18) \\ \text { C16 } & 0.059(2) & 0.059(2) & 0.045(2) & -0.0130(18) & 0.0163(18) & -0.0120(17) \\ \text { C17 } & 0.0442(19) & 0.0403(16) & 0.0381(18) & -0.0054(14) & 0.0107(15) & 0.0023(14) \\ \text { C18 } & 0.0422(19) & 0.0401(16) & 0.0425(19) & 0.0005(14) & 0.0156(15) & 0.0092(14) \\ \text { C19 } & 0.058(2) & 0.0480(19) & 0.052(2) & -0.0087(17) & 0.0170(19) & 0.0094(17) \\ \text { C20 } & 0.061(2) & 0.055(2) & 0.064(3) & -0.0059(18) & 0.026(2) & 0.0199(19) \\ \text { C21 } & 0.070(3) & 0.052(2) & 0.054(2) & 0.0074(19) & 0.034(2) & 0.0134(18) \\ \text { C22 } & 0.068(2) & 0.0470(19) & 0.046(2) & 0.0061(17) & 0.0270(19) & 0.0053(16) \\ \text { C23 } & 0.048(2) & 0.0387(16) & 0.0425(19) & 0.0098(14) & 0.0174(16) & 0.0082(14) \\ \text { C24 } & 0.139(5) & 0.113(4) & 0.066(3) & -0.090(4) & 0.013(3) & 0.008(3) \\ & & & & & & \end{array}$

Geometric parameters $\left(\AA,{ }^{\circ}\right)$

\begin{tabular}{llll}
\hline $\mathrm{Ni} 1-\mathrm{O} 3$ & $1.832(2)$ & $\mathrm{C} 9-\mathrm{C} 14$ & $1.391(4)$ \\
$\mathrm{N} 11-\mathrm{O} 1$ & $1.833(2)$ & $\mathrm{C} 10-\mathrm{C} 11$ & $1.382(4)$ \\
$\mathrm{N} 1-\mathrm{N} 1$ & $1.845(2)$ & $\mathrm{C} 10-\mathrm{H} 10$ & 0.9300 \\
$\mathrm{~N} 11-\mathrm{N} 2$ & $1.848(2)$ & $\mathrm{C} 11-\mathrm{C} 12$ & $1.402(4)$ \\
$\mathrm{O} 1-\mathrm{C} 1$ & $1.299(4)$ & $\mathrm{C} 11-\mathrm{C} 15$ & $1.509(4)$ \\
$\mathrm{O} 2-\mathrm{C} 5$ & $1.367(4)$ & $\mathrm{C} 12-\mathrm{C} 13$ & $1.380(4)$ \\
$\mathrm{O} 2-\mathrm{C} 7$ & $1.428(4)$ & $\mathrm{C} 12-\mathrm{C} 16$ & $1.509(4)$ \\
$\mathrm{O} 3-\mathrm{C} 23$ & $1.297(4)$ & $\mathrm{C} 13-\mathrm{C} 14$ & $1.392(4)$ \\
$\mathrm{O} 4-\mathrm{C} 19$ & $1.358(4)$ & $\mathrm{C} 13-\mathrm{H} 13$ & 0.9300 \\
$\mathrm{O} 4-\mathrm{C} 24$ & $1.438(4)$ & $\mathrm{C} 15-\mathrm{H} 15 \mathrm{~A}$ & 0.9600 \\
$\mathrm{~N} 1-\mathrm{C} 8$ & $1.313(4)$ & $\mathrm{C} 15-\mathrm{H} 15 \mathrm{~B}$ & 0.9600 \\
$\mathrm{~N} 1-\mathrm{C} 9$ & $1.430(4)$ & $\mathrm{C} 15-\mathrm{H} 15 \mathrm{C}$ & 0.9600 \\
$\mathrm{~N} 2-\mathrm{C} 17$ & $1.302(4)$ & $\mathrm{C} 16-\mathrm{H} 16 \mathrm{~A}$ & 0.9600 \\
$\mathrm{~N} 2-\mathrm{C} 14$ & $1.433(4)$ & $\mathrm{C} 16-\mathrm{H} 16 \mathrm{~B}$ & 0.9600 \\
$\mathrm{C} 1-\mathrm{C} 2$ & $1.410(4)$ & $\mathrm{C} 16-\mathrm{H} 16 \mathrm{C}$ & 0.9600 \\
$\mathrm{C} 1-\mathrm{C} 6$ & $1.419(4)$ & $\mathrm{C} 17-\mathrm{C} 18$ & $1.411(4)$ \\
$\mathrm{C} 2-\mathrm{C} 3$ & $1.365(5)$ & $\mathrm{C} 17-\mathrm{H} 17$ & 0.9300 \\
$\mathrm{C} 2-\mathrm{H} 2$ & 0.9300 & $\mathrm{C} 18-\mathrm{C} 23$ & $1.415(4)$ \\
$\mathrm{C} 3-\mathrm{C} 4$ & $1.395(5)$ & $\mathrm{C} 18-\mathrm{C} 19$ & $1.434(4)$ \\
$\mathrm{C} 3-\mathrm{H} 3$ & 0.9300 & $\mathrm{C} 19-\mathrm{C} 20$ & $1.363(5)$ \\
$\mathrm{C} 4-\mathrm{C} 5$ & $1.361(4)$ & $\mathrm{C} 20-\mathrm{C} 21$ & $1.390(5)$
\end{tabular}




\begin{tabular}{|c|c|c|c|}
\hline $\mathrm{C} 4-\mathrm{H} 4$ & 0.9300 & $\mathrm{C} 20-\mathrm{H} 20$ & 0.9300 \\
\hline $\mathrm{C} 5-\mathrm{C} 6$ & $1.437(4)$ & $\mathrm{C} 21-\mathrm{C} 22$ & $1.364(5)$ \\
\hline $\mathrm{C} 6-\mathrm{C} 8$ & $1.407(4)$ & $\mathrm{C} 21-\mathrm{H} 21$ & 0.9300 \\
\hline C7-H7A & 0.9600 & $\mathrm{C} 22-\mathrm{C} 23$ & $1.409(4)$ \\
\hline C7-H7B & 0.9600 & $\mathrm{C} 22-\mathrm{H} 22$ & 0.9300 \\
\hline $\mathrm{C} 7-\mathrm{H} 7 \mathrm{C}$ & 0.9600 & $\mathrm{C} 24-\mathrm{H} 24 \mathrm{~A}$ & 0.9600 \\
\hline $\mathrm{C} 8-\mathrm{H} 8$ & 0.9300 & $\mathrm{C} 24-\mathrm{H} 24 \mathrm{~B}$ & 0.9600 \\
\hline $\mathrm{C} 9-\mathrm{C} 10$ & $1.382(4)$ & $\mathrm{C} 24-\mathrm{H} 24 \mathrm{C}$ & 0.9600 \\
\hline $\mathrm{O} 3-\mathrm{Ni} 1-\mathrm{O} 1$ & $83.87(9)$ & $\mathrm{C} 10-\mathrm{C} 11-\mathrm{C} 15$ & $119.5(3)$ \\
\hline $\mathrm{O} 3-\mathrm{Ni1}-\mathrm{N} 1$ & $177.97(11)$ & $\mathrm{C} 12-\mathrm{C} 11-\mathrm{C} 15$ & $121.0(3)$ \\
\hline $\mathrm{O} 1-\mathrm{Ni} 1-\mathrm{N} 1$ & $94.67(10)$ & $\mathrm{C} 13-\mathrm{C} 12-\mathrm{C} 11$ & $119.4(3)$ \\
\hline $\mathrm{O} 3-\mathrm{Ni} 1-\mathrm{N} 2$ & $94.57(10)$ & $\mathrm{C} 13-\mathrm{C} 12-\mathrm{C} 16$ & $119.7(3)$ \\
\hline $\mathrm{O} 1-\mathrm{Ni1}-\mathrm{N} 2$ & $178.42(10)$ & $\mathrm{C} 11-\mathrm{C} 12-\mathrm{C} 16$ & $120.8(3)$ \\
\hline $\mathrm{N} 1-\mathrm{Ni1}-\mathrm{N} 2$ & $86.88(10)$ & $\mathrm{C} 12-\mathrm{C} 13-\mathrm{C} 14$ & $120.9(3)$ \\
\hline $\mathrm{C} 1-\mathrm{O} 1-\mathrm{Ni} 1$ & $128.5(2)$ & $\mathrm{C} 12-\mathrm{C} 13-\mathrm{H} 13$ & 119.6 \\
\hline $\mathrm{C} 5-\mathrm{O} 2-\mathrm{C} 7$ & $117.1(3)$ & $\mathrm{C} 14-\mathrm{C} 13-\mathrm{H} 13$ & 119.6 \\
\hline $\mathrm{C} 23-\mathrm{O} 3-\mathrm{Ni} 1$ & $128.4(2)$ & $\mathrm{C} 9-\mathrm{C} 14-\mathrm{C} 13$ & $119.4(3)$ \\
\hline $\mathrm{C} 19-\mathrm{O} 4-\mathrm{C} 24$ & $116.8(3)$ & $\mathrm{C} 9-\mathrm{C} 14-\mathrm{N} 2$ & $113.6(3)$ \\
\hline $\mathrm{C} 8-\mathrm{N} 1-\mathrm{C} 9$ & $120.8(3)$ & $\mathrm{C} 13-\mathrm{C} 14-\mathrm{N} 2$ & $127.0(3)$ \\
\hline $\mathrm{C} 8-\mathrm{N} 1-\mathrm{Ni} 1$ & $126.2(2)$ & $\mathrm{C} 11-\mathrm{C} 15-\mathrm{H} 15 \mathrm{~A}$ & 109.5 \\
\hline $\mathrm{C} 9-\mathrm{N} 1-\mathrm{Ni1}$ & $112.93(18)$ & $\mathrm{C} 11-\mathrm{C} 15-\mathrm{H} 15 \mathrm{~B}$ & 109.5 \\
\hline $\mathrm{C} 17-\mathrm{N} 2-\mathrm{C} 14$ & $121.3(3)$ & $\mathrm{H} 15 \mathrm{~A}-\mathrm{C} 15-\mathrm{H} 15 \mathrm{~B}$ & 109.5 \\
\hline $\mathrm{C} 17-\mathrm{N} 2-\mathrm{Ni1}$ & $125.9(2)$ & $\mathrm{C} 11-\mathrm{C} 15-\mathrm{H} 15 \mathrm{C}$ & 109.5 \\
\hline $\mathrm{C} 14-\mathrm{N} 2-\mathrm{Ni} 1$ & $112.73(18)$ & $\mathrm{H} 15 \mathrm{~A}-\mathrm{C} 15-\mathrm{H} 15 \mathrm{C}$ & 109.5 \\
\hline $\mathrm{O} 1-\mathrm{C} 1-\mathrm{C} 2$ & $118.1(3)$ & $\mathrm{H} 15 \mathrm{~B}-\mathrm{C} 15-\mathrm{H} 15 \mathrm{C}$ & 109.5 \\
\hline $\mathrm{O} 1-\mathrm{C} 1-\mathrm{C} 6$ & $123.4(3)$ & $\mathrm{C} 12-\mathrm{C} 16-\mathrm{H} 16 \mathrm{~A}$ & 109.5 \\
\hline $\mathrm{C} 2-\mathrm{C} 1-\mathrm{C} 6$ & $118.5(3)$ & $\mathrm{C} 12-\mathrm{C} 16-\mathrm{H} 16 \mathrm{~B}$ & 109.5 \\
\hline $\mathrm{C} 3-\mathrm{C} 2-\mathrm{C} 1$ & $120.4(3)$ & $\mathrm{H} 16 \mathrm{~A}-\mathrm{C} 16-\mathrm{H} 16 \mathrm{~B}$ & 109.5 \\
\hline $\mathrm{C} 3-\mathrm{C} 2-\mathrm{H} 2$ & 119.8 & $\mathrm{C} 12-\mathrm{C} 16-\mathrm{H} 16 \mathrm{C}$ & 109.5 \\
\hline $\mathrm{C} 1-\mathrm{C} 2-\mathrm{H} 2$ & 119.8 & $\mathrm{H} 16 \mathrm{~A}-\mathrm{C} 16-\mathrm{H} 16 \mathrm{C}$ & 109.5 \\
\hline $\mathrm{C} 2-\mathrm{C} 3-\mathrm{C} 4$ & $122.2(3)$ & $\mathrm{H} 16 \mathrm{~B}-\mathrm{C} 16-\mathrm{H} 16 \mathrm{C}$ & 109.5 \\
\hline $\mathrm{C} 2-\mathrm{C} 3-\mathrm{H} 3$ & 118.9 & $\mathrm{~N} 2-\mathrm{C} 17-\mathrm{C} 18$ & $125.4(3)$ \\
\hline $\mathrm{C} 4-\mathrm{C} 3-\mathrm{H} 3$ & 118.9 & $\mathrm{~N} 2-\mathrm{C} 17-\mathrm{H} 17$ & 117.3 \\
\hline $\mathrm{C} 5-\mathrm{C} 4-\mathrm{C} 3$ & $119.0(3)$ & $\mathrm{C} 18-\mathrm{C} 17-\mathrm{H} 17$ & 117.3 \\
\hline $\mathrm{C} 5-\mathrm{C} 4-\mathrm{H} 4$ & 120.5 & $\mathrm{C} 17-\mathrm{C} 18-\mathrm{C} 23$ & $121.5(3)$ \\
\hline $\mathrm{C} 3-\mathrm{C} 4-\mathrm{H} 4$ & 120.5 & $\mathrm{C} 17-\mathrm{C} 18-\mathrm{C} 19$ & $120.0(3)$ \\
\hline $\mathrm{C} 4-\mathrm{C} 5-\mathrm{O} 2$ & $124.2(3)$ & $\mathrm{C} 23-\mathrm{C} 18-\mathrm{C} 19$ & $118.5(3)$ \\
\hline $\mathrm{C} 4-\mathrm{C} 5-\mathrm{C} 6$ & $121.0(3)$ & $\mathrm{O} 4-\mathrm{C} 19-\mathrm{C} 20$ & $124.9(3)$ \\
\hline $\mathrm{O} 2-\mathrm{C} 5-\mathrm{C} 6$ & $114.7(3)$ & $\mathrm{O} 4-\mathrm{C} 19-\mathrm{C} 18$ & $113.9(3)$ \\
\hline $\mathrm{C} 8-\mathrm{C} 6-\mathrm{C} 1$ & $121.7(3)$ & $\mathrm{C} 20-\mathrm{C} 19-\mathrm{C} 18$ & $121.2(3)$ \\
\hline $\mathrm{C} 8-\mathrm{C} 6-\mathrm{C} 5$ & $119.4(3)$ & $\mathrm{C} 19-\mathrm{C} 20-\mathrm{C} 21$ & $119.1(3)$ \\
\hline $\mathrm{C} 1-\mathrm{C} 6-\mathrm{C} 5$ & $118.8(3)$ & $\mathrm{C} 19-\mathrm{C} 20-\mathrm{H} 20$ & 120.4 \\
\hline $\mathrm{O} 2-\mathrm{C} 7-\mathrm{H} 7 \mathrm{~A}$ & 109.5 & $\mathrm{C} 21-\mathrm{C} 20-\mathrm{H} 20$ & 120.4 \\
\hline $\mathrm{O} 2-\mathrm{C} 7-\mathrm{H} 7 \mathrm{~B}$ & 109.5 & $\mathrm{C} 22-\mathrm{C} 21-\mathrm{C} 20$ & $122.1(3)$ \\
\hline $\mathrm{H} 7 \mathrm{~A}-\mathrm{C} 7-\mathrm{H} 7 \mathrm{~B}$ & 109.5 & $\mathrm{C} 22-\mathrm{C} 21-\mathrm{H} 21$ & 119.0 \\
\hline $\mathrm{O} 2-\mathrm{C} 7-\mathrm{H} 7 \mathrm{C}$ & 109.5 & $\mathrm{C} 20-\mathrm{C} 21-\mathrm{H} 21$ & 119.0 \\
\hline $\mathrm{H} 7 \mathrm{~A}-\mathrm{C} 7-\mathrm{H} 7 \mathrm{C}$ & 109.5 & $\mathrm{C} 21-\mathrm{C} 22-\mathrm{C} 23$ & $120.4(4)$ \\
\hline
\end{tabular}




\begin{tabular}{|c|c|c|c|}
\hline $\mathrm{H} 7 \mathrm{~B}-\mathrm{C} 7-\mathrm{H} 7 \mathrm{C}$ & 109.5 & $\mathrm{C} 21-\mathrm{C} 22-\mathrm{H} 22$ & 119.8 \\
\hline $\mathrm{N} 1-\mathrm{C} 8-\mathrm{C} 6$ & $125.1(3)$ & $\mathrm{C} 23-\mathrm{C} 22-\mathrm{H} 22$ & 119.8 \\
\hline $\mathrm{N} 1-\mathrm{C} 8-\mathrm{H} 8$ & 117.5 & $\mathrm{O} 3-\mathrm{C} 23-\mathrm{C} 22$ & $118.0(3)$ \\
\hline $\mathrm{C} 6-\mathrm{C} 8-\mathrm{H} 8$ & 117.5 & $\mathrm{O} 3-\mathrm{C} 23-\mathrm{C} 18$ & $123.3(3)$ \\
\hline $\mathrm{C} 10-\mathrm{C} 9-\mathrm{C} 14$ & $119.8(3)$ & $\mathrm{C} 22-\mathrm{C} 23-\mathrm{C} 18$ & $118.7(3)$ \\
\hline $\mathrm{C} 10-\mathrm{C} 9-\mathrm{N} 1$ & $126.5(3)$ & $\mathrm{O} 4-\mathrm{C} 24-\mathrm{H} 24 \mathrm{~A}$ & 109.5 \\
\hline $\mathrm{C} 14-\mathrm{C} 9-\mathrm{N} 1$ & $113.7(3)$ & $\mathrm{O} 4-\mathrm{C} 24-\mathrm{H} 24 \mathrm{~B}$ & 109.5 \\
\hline $\mathrm{C} 9-\mathrm{C} 10-\mathrm{C} 11$ & $120.9(3)$ & $\mathrm{H} 24 \mathrm{~A}-\mathrm{C} 24-\mathrm{H} 24 \mathrm{~B}$ & 109.5 \\
\hline $\mathrm{C} 9-\mathrm{C} 10-\mathrm{H} 10$ & 119.5 & $\mathrm{O} 4-\mathrm{C} 24-\mathrm{H} 24 \mathrm{C}$ & 109.5 \\
\hline $\mathrm{C} 11-\mathrm{C} 10-\mathrm{H} 10$ & 119.5 & $\mathrm{H} 24 \mathrm{~A}-\mathrm{C} 24-\mathrm{H} 24 \mathrm{C}$ & 109.5 \\
\hline $\mathrm{C} 10-\mathrm{C} 11-\mathrm{C} 12$ & $119.5(3)$ & $\mathrm{H} 24 \mathrm{~B}-\mathrm{C} 24-\mathrm{H} 24 \mathrm{C}$ & 109.5 \\
\hline $\mathrm{O} 3-\mathrm{Ni1}-\mathrm{O} 1-\mathrm{C} 1$ & $179.7(3)$ & $\mathrm{C} 9-\mathrm{C} 10-\mathrm{C} 11-\mathrm{C} 12$ & $-0.8(5)$ \\
\hline $\mathrm{N} 1-\mathrm{Ni} 1-\mathrm{O} 1-\mathrm{C} 1$ & $1.2(3)$ & $\mathrm{C} 9-\mathrm{C} 10-\mathrm{C} 11-\mathrm{C} 15$ & $179.3(3)$ \\
\hline $\mathrm{O} 1-\mathrm{Ni1}-\mathrm{O} 3-\mathrm{C} 23$ & $173.2(3)$ & $\mathrm{C} 10-\mathrm{C} 11-\mathrm{C} 12-\mathrm{C} 13$ & $-0.4(5)$ \\
\hline $\mathrm{N} 2-\mathrm{Ni} 1-\mathrm{O} 3-\mathrm{C} 23$ & $-7.1(3)$ & $\mathrm{C} 15-\mathrm{C} 11-\mathrm{C} 12-\mathrm{C} 13$ & $179.5(3)$ \\
\hline $\mathrm{O} 1-\mathrm{Ni} 1-\mathrm{N} 1-\mathrm{C} 8$ & $-5.6(3)$ & $\mathrm{C} 10-\mathrm{C} 11-\mathrm{C} 12-\mathrm{C} 16$ & $176.5(3)$ \\
\hline $\mathrm{N} 2-\mathrm{Ni} 1-\mathrm{N} 1-\mathrm{C} 8$ & $174.6(3)$ & $\mathrm{C} 15-\mathrm{C} 11-\mathrm{C} 12-\mathrm{C} 16$ & $-3.6(5)$ \\
\hline $\mathrm{O} 1-\mathrm{Ni} 1-\mathrm{N} 1-\mathrm{C} 9$ & $175.77(19)$ & $\mathrm{C} 11-\mathrm{C} 12-\mathrm{C} 13-\mathrm{C} 14$ & $1.1(5)$ \\
\hline $\mathrm{N} 2-\mathrm{Ni} 1-\mathrm{N} 1-\mathrm{C} 9$ & $-4.0(2)$ & $\mathrm{C} 16-\mathrm{C} 12-\mathrm{C} 13-\mathrm{C} 14$ & $-175.8(3)$ \\
\hline $\mathrm{O} 3-\mathrm{Ni1}-\mathrm{N} 2-\mathrm{C} 17$ & $8.2(3)$ & $\mathrm{C} 10-\mathrm{C} 9-\mathrm{C} 14-\mathrm{C} 13$ & $-0.6(4)$ \\
\hline $\mathrm{N} 1-\mathrm{Ni1}-\mathrm{N} 2-\mathrm{C} 17$ & $-173.2(3)$ & $\mathrm{N} 1-\mathrm{C} 9-\mathrm{C} 14-\mathrm{C} 13$ & $178.5(3)$ \\
\hline $\mathrm{O} 3-\mathrm{Ni} 1-\mathrm{N} 2-\mathrm{C} 14$ & $-174.79(19)$ & $\mathrm{C} 10-\mathrm{C} 9-\mathrm{C} 14-\mathrm{N} 2$ & $-179.4(3)$ \\
\hline $\mathrm{N} 1-\mathrm{Ni1}-\mathrm{N} 2-\mathrm{C} 14$ & $3.8(2)$ & $\mathrm{N} 1-\mathrm{C} 9-\mathrm{C} 14-\mathrm{N} 2$ & $-0.3(4)$ \\
\hline $\mathrm{Ni} 1-\mathrm{O} 1-\mathrm{C} 1-\mathrm{C} 2$ & $-176.2(2)$ & $\mathrm{C} 12-\mathrm{C} 13-\mathrm{C} 14-\mathrm{C} 9$ & $-0.6(5)$ \\
\hline $\mathrm{Ni} 1-\mathrm{O} 1-\mathrm{C} 1-\mathrm{C} 6$ & $3.6(5)$ & $\mathrm{C} 12-\mathrm{C} 13-\mathrm{C} 14-\mathrm{N} 2$ & $177.9(3)$ \\
\hline $\mathrm{O} 1-\mathrm{C} 1-\mathrm{C} 2-\mathrm{C} 3$ & $-179.7(3)$ & $\mathrm{C} 17-\mathrm{N} 2-\mathrm{C} 14-\mathrm{C} 9$ & $174.4(3)$ \\
\hline $\mathrm{C} 6-\mathrm{C} 1-\mathrm{C} 2-\mathrm{C} 3$ & $0.4(5)$ & $\mathrm{Ni1}-\mathrm{N} 2-\mathrm{C} 14-\mathrm{C} 9$ & $-2.8(3)$ \\
\hline $\mathrm{C} 1-\mathrm{C} 2-\mathrm{C} 3-\mathrm{C} 4$ & $1.2(6)$ & $\mathrm{C} 17-\mathrm{N} 2-\mathrm{C} 14-\mathrm{C} 13$ & $-4.3(5)$ \\
\hline $\mathrm{C} 2-\mathrm{C} 3-\mathrm{C} 4-\mathrm{C} 5$ & $-1.1(5)$ & $\mathrm{Ni1}-\mathrm{N} 2-\mathrm{C} 14-\mathrm{C} 13$ & $178.5(3)$ \\
\hline $\mathrm{C} 3-\mathrm{C} 4-\mathrm{C} 5-\mathrm{O} 2$ & $-178.6(3)$ & $\mathrm{C} 14-\mathrm{N} 2-\mathrm{C} 17-\mathrm{C} 18$ & $179.8(3)$ \\
\hline $\mathrm{C} 3-\mathrm{C} 4-\mathrm{C} 5-\mathrm{C} 6$ & $-0.7(5)$ & Ni1-N2-C17-C18 & $-3.4(5)$ \\
\hline $\mathrm{C} 7-\mathrm{O} 2-\mathrm{C} 5-\mathrm{C} 4$ & $6.2(5)$ & $\mathrm{N} 2-\mathrm{C} 17-\mathrm{C} 18-\mathrm{C} 23$ & $-5.6(5)$ \\
\hline $\mathrm{C} 7-\mathrm{O} 2-\mathrm{C} 5-\mathrm{C} 6$ & $-171.8(3)$ & $\mathrm{N} 2-\mathrm{C} 17-\mathrm{C} 18-\mathrm{C} 19$ & $177.4(3)$ \\
\hline $\mathrm{O} 1-\mathrm{C} 1-\mathrm{C} 6-\mathrm{C} 8$ & $-5.1(5)$ & $\mathrm{C} 24-\mathrm{O} 4-\mathrm{C} 19-\mathrm{C} 20$ & $-1.2(6)$ \\
\hline $\mathrm{C} 2-\mathrm{C} 1-\mathrm{C} 6-\mathrm{C} 8$ & $174.8(3)$ & $\mathrm{C} 24-\mathrm{O} 4-\mathrm{C} 19-\mathrm{C} 18$ & $179.3(4)$ \\
\hline $\mathrm{O} 1-\mathrm{C} 1-\mathrm{C} 6-\mathrm{C} 5$ & $178.1(3)$ & $\mathrm{C} 17-\mathrm{C} 18-\mathrm{C} 19-\mathrm{O} 4$ & $-5.8(5)$ \\
\hline $\mathrm{C} 2-\mathrm{C} 1-\mathrm{C} 6-\mathrm{C} 5$ & $-2.1(4)$ & $\mathrm{C} 23-\mathrm{C} 18-\mathrm{C} 19-\mathrm{O} 4$ & $177.2(3)$ \\
\hline $\mathrm{C} 4-\mathrm{C} 5-\mathrm{C} 6-\mathrm{C} 8$ & $-174.6(3)$ & $\mathrm{C} 17-\mathrm{C} 18-\mathrm{C} 19-\mathrm{C} 20$ & $174.7(3)$ \\
\hline $\mathrm{O} 2-\mathrm{C} 5-\mathrm{C} 6-\mathrm{C} 8$ & $3.4(4)$ & $\mathrm{C} 23-\mathrm{C} 18-\mathrm{C} 19-\mathrm{C} 20$ & $-2.3(5)$ \\
\hline $\mathrm{C} 4-\mathrm{C} 5-\mathrm{C} 6-\mathrm{C} 1$ & $2.3(5)$ & $\mathrm{O} 4-\mathrm{C} 19-\mathrm{C} 20-\mathrm{C} 21$ & $-179.5(4)$ \\
\hline $\mathrm{O} 2-\mathrm{C} 5-\mathrm{C} 6-\mathrm{C} 1$ & $-179.7(3)$ & $\mathrm{C} 18-\mathrm{C} 19-\mathrm{C} 20-\mathrm{C} 21$ & $-0.1(6)$ \\
\hline $\mathrm{C} 9-\mathrm{N} 1-\mathrm{C} 8-\mathrm{C} 6$ & $-175.9(3)$ & $\mathrm{C} 19-\mathrm{C} 20-\mathrm{C} 21-\mathrm{C} 22$ & $1.3(6)$ \\
\hline $\mathrm{Ni} 1-\mathrm{N} 1-\mathrm{C} 8-\mathrm{C} 6$ & $5.6(4)$ & $\mathrm{C} 20-\mathrm{C} 21-\mathrm{C} 22-\mathrm{C} 23$ & $-0.1(5)$ \\
\hline $\mathrm{C} 1-\mathrm{C} 6-\mathrm{C} 8-\mathrm{N} 1$ & $0.3(5)$ & $\mathrm{Ni} 1-\mathrm{O} 3-\mathrm{C} 23-\mathrm{C} 22$ & $-178.7(2)$ \\
\hline $\mathrm{C} 5-\mathrm{C} 6-\mathrm{C} 8-\mathrm{N} 1$ & $177.1(3)$ & $\mathrm{Ni1}-\mathrm{O} 3-\mathrm{C} 23-\mathrm{C} 18$ & $0.8(4)$ \\
\hline $\mathrm{C} 8-\mathrm{N} 1-\mathrm{C} 9-\mathrm{C} 10$ & $3.6(5)$ & $\mathrm{C} 21-\mathrm{C} 22-\mathrm{C} 23-\mathrm{O} 3$ & $177.2(3)$ \\
\hline $\mathrm{Ni1}-\mathrm{N} 1-\mathrm{C} 9-\mathrm{C} 10$ & $-177.7(3)$ & $\mathrm{C} 21-\mathrm{C} 22-\mathrm{C} 23-\mathrm{C} 18$ & $-2.4(5)$ \\
\hline
\end{tabular}




\begin{tabular}{llll}
$\mathrm{C} 8-\mathrm{N} 1-\mathrm{C} 9-\mathrm{C} 14$ & $-175.4(3)$ & $\mathrm{C} 17-\mathrm{C} 18-\mathrm{C} 23-\mathrm{O} 3$ & $7.0(5)$ \\
$\mathrm{N} 11-\mathrm{N} 1-\mathrm{C} 9-\mathrm{C} 14$ & $3.3(3)$ & $\mathrm{C} 19-\mathrm{C} 18-\mathrm{C} 23-\mathrm{O} 3$ & $-176.0(3)$ \\
$\mathrm{C} 14-\mathrm{C} 9-\mathrm{C} 10-\mathrm{C} 11$ & $1.3(5)$ & $\mathrm{C} 17-\mathrm{C} 18-\mathrm{C} 23-\mathrm{C} 22$ & $-173.5(3)$ \\
$\mathrm{N} 1-\mathrm{C} 9-\mathrm{C} 10-\mathrm{C} 11$ & $-177.6(3)$ & $\mathrm{C} 19-\mathrm{C} 18-\mathrm{C} 23-\mathrm{C} 22$ & $3.5(5)$ \\
\hline
\end{tabular}

Hydrogen-bond geometry $\left(\AA,{ }^{\circ}\right)$

\begin{tabular}{lllll}
\hline$D-\mathrm{H}^{\cdots} \cdots A$ & $D-\mathrm{H}$ & $\mathrm{H} \cdots A$ & $D \cdots A$ & $D-\mathrm{H}^{\cdots} \cdots A$ \\
\hline $\mathrm{C} 7-\mathrm{H} 7 C \cdots{ }^{\mathrm{O}} 3^{\mathrm{i}}$ & 0.96 & 2.51 & $3.424(5)$ & 158 \\
$\mathrm{C} 21-\mathrm{H} 21 \cdots 2^{\mathrm{ii}}$ & 0.93 & 2.52 & $3.340(4)$ & 147 \\
\hline
\end{tabular}

Symmetry codes: (i) $-x+1 / 2, y+1 / 2,-z+1 / 2$; (ii) $x-1 / 2,-y+3 / 2, z+1 / 2$. 